\title{
NOTES OF A CASE
}

\section{or \\ MERCURIAL ERETHISM.}

By T. BATEMAN, M.D. F.R.S. \&ic.

\section{Read March 31, 1818.}

THE singular and often fatal affection which occasionally supervenes during a course of Mercury, when that medicine acts as a poison on the system, was first noticed, with the above appellation, by that able pathologist, Mr. John Pearson; and his terse and perspicuous description of the symptoms which characterize it, constitutes, I believe, the only history of the disease to be found in his medical ,writings*. It is evident, however, that the features of the malady are not sufficiently known, even to the most enlightened members of the profession : for the failure on the part of the medical , advisers, in the instance about to be related, to recognise its first symptoms, and the consequent repetition of the dose of the poison, after

* See his Observations on the effects of various articles of the Mat. Med. in Lues Venerea. Chap. XII. 
its commencement, had nearly rendered it fatal. I conceive, therefore, that a narrative of the progress of a severe case of this disease may not be uninteresting to the Society, and that by affording a more distinct view of its character, it may perhaps contribute to preserve other individuals from the sufferings and danger incurred in this instance.

The mercurial inunction which occasioned this erethism, was resorted to under the sanction of the first medical authority, with a view to relieve an amaurosis of the right eye, which had come on during the preceding summer, in connection with a considerable derangement of the chylopoetic functions, and was commenced after a residence of three months at Brighton, during which these functions and the general health had been materially improved.

\section{CASE.}

January 16th, 1817. Began mercurial friction, using a drachm of the ointment every night.

23rd. Gums slightly tender; nightly febricula.

24th. Languid and feverish; gums reddish and slightly spongy. At four in the morning of the 25th, a violent and irregular beating of the heart came on, which did not yield to laudanum and stimulants, but suddenly went off about one, P.M. 
25th. A very feverish night, with much perspiration, and early in the morning a copious motion with much griping, a Seidlitz powder taken the preceding morning having failed to operate $m$ the course of the day.

26th. On getting up, very severe griping, which continued nearly all day, without tenesmus, but somewhat relieved towards evening by opiate lozenges; mouth more decidedly sore, and irregularity of circulation returning at 11 A.M. and continuing all day, omitted the mercury this night.

27th. Palpitation went off during sleep last night, and the feelings being comfortable during the day, the friction was repeated at night; but towards morning the heart's action became again distarbed.

Tuesday, 28th. As this disturbance continued, it was determined at a consultation this afternoon, that it was absolutely necessary to desist altogether from the use of mercury at present: During the remainder of this week, the disturbed action of the heart continuing unremitted, the strength and power of exertion became considerably impaired, yet the patient was able to walk some distance, and rode a good deal in coaches, and on Friday and Saturday passed the evening in visits to two friends. During this week, a cough, which had been slightly troublesome for some time, and which was obviously connected with a flatulent state of 
the stomach, and not of pulmonary origin, increased very much, coming on in violent paroxysms, and producing retchings, though not vomiting. These paroxysms were generally relieved by spiced wine, taken hot, in small quantities; which was now deemed necessary as a cordial.

Sunday, February 2d. Got up with a considerable increase of languor, having passed a feverish and almost sleepless night, and from this time was unable to leave the house.

This languor and debility continued to increase rapidly every day, as well as the cough, which was excessively harassing, and produced a distressing sense of binding and of immovable constriction across the region of the diaphragm; which; together with the continuing perturbation of the heart, rendered it necessary to remain in nearly an upright posture even during the night. So early as Wednesday the $5 \mathrm{th}$, the depression had become very great; but a slight degree of vigour appeared to be restored on the following day by a more liberal use of spiced wine, a bottle of which was taken in the ccurse of twenty-four hours, it having been found necessary to use it even during the night. The nights were now passed almost entirely without sleep, in consequence of the disturbed state of the circulation, and the constant harassing spasmodic cough, as well as from the distress 
occasioned by painful distension of the stomact by flatulence.

Friday, 7th. On this night, from the difficulty of being supported in an erect posture in bed, and from a dread of the sufferings produced by any approximation to a supine position, or to lying on either side, it was determined to pass the night propped up on a couch in the drawing-room. No sleep was obtained, but in the course of the night the action of the heart became once more regular, in consequence of which the following day (the 8th) was passed with less suffering. On taking the nightly position on the couch this night, fell asleep, and slept quietly about an hour; awoke with a start and with a momentary confusion of mind, at the same time calling out, in apparent distress, for the admission of fresh air; on the. return of consciousness, the irregular action of the heart was found to have recommenced. The extract of hyoscyamus, which had been taken on Thursday and Friday, the 6th and 7th, in doses, first of three, and then of five grains, was supposed to have quieted the action of the heart, and to have contributed to the intermission of Saturday; but it was taken three times during the night of the 8 th, in doses of five grains each, without any obvious effect. The tincture of hop was then substituted and taken in doses of thirty drops every six hours until the 12th, without any other effect 
than the production of a slight drowsiness, which at length became considerable. Having found for some days past that the languor and debility were much increased in the morning, and obviously diminished by frequent supplies of food and cordials during the day, it was now deemed advisable to continue them during the night. This plan was begun on Wednesday the 5th; and on Sunday the 9 th, the languor and debility being much in. creased after the return of the irregularity of the circulation; these supplies were ordered to be repeated every hour during the day and night; con. sisting of small quantities of jelly, beef-tea with rusks, chicken, hare, milk, \&zc. and the stimulant quality of the spiced wine was increased by the addition of a tea-spoonful - of brandy, which was also occasionally taken alone, diluted with water. On going to the couch this night, a disturbed sleep of only a quarter of an hour was again terminated by a start and confusion, and by a still more importunate demand of fresh air; which was not satisfied till two windows and the door were set open, and the couch drawn into the current. No more sleep was obtained, and one window and the door were kept open till morning. The two following nights were passed without sleep, and with some violent paroxysms of coughing and retching, which were generally somewhat relieved by food and cordials; the admission' of air being also from time to time necessary. On the night of Wednesday the 12th, having again obtained a short sleep, not 
axceeding a quarter of an hour, awoke in extreme distress, with a sensation of sinking, which was felt as that of approaching dissolution; the anxious demand for fresh air was repeated, as well as urgent calls for brandy undiluted, which was greedily swallowed to the extent of three glasses in the course of five minutes, without producing much relief. Ammonia and pther were then substituted, one or other of which was repeated every ten minutes for about two hours, when the faintness rapidly declined, especially after a very copious discharge of limpid urine, During this paroxysm, notwithstanding the total depression of muscular power, and the feeling of sinking to immediate death, the mental powers remained clear, the extremities warm, and the pulse, though extremely irregular, and not acconding exaetly in its beats with the contractions of the heart, was felt in all the extremities. About two hour afterwards, without any previous sleep, and although the wipdows apd doors had remained open, a second and similar paroxysin of fainting oecurred, in which the appearance of depression was greater, at least in the power of speaking, and which, after a similar administration of stimulants, gradually subsided in the course of two hours leaving hawever a much greater degree of langupr and depression than the former. It was now distinctly abvious, that the action of the beart and arteries, which was extremely foeble as well as irregular while awake, was so much more enfoebled during 
sleep, as to be in fact almost suspended, and thus to occasion those alarming faintings and sinkings; so that it became necessary, notwithstanding the extreme drowsiness which had succeeded the long continued watchfulness, to interrupt the sleep at the expiration of two minutes, by which time, or even sooner, the sinking of the pulse and countenance indicated the approaching languor.

Thursday; 13th. The debility left by the two paroxysms of the preceding night was extreme, and it was still necessary both day and night to watch and interrupt the sleep every two minutes; this measure was necessarily continued for three weeks or more, during which period the length of the permitted slumbers was gradually increased, having reached five minutes in eight or nine days, and soon afterwards a quarter of an hour, still however producing similar paroxysms of fainting, more or less severe. The distress, indeed, accompanying these paroxysms, excited so much dread of indulging the overpowering drawsiness, that the most urgent requests were made by the patient for strictly watching and interrupting the sleep. In this state of debility the powers of the stomach seemed to fail rapidly; it became incapable of recoiving the solid animal diet which had been hitherto so much relished. During the whole of this day and night, beef-tea only was used, a few spoonfuls being taken every half hour, sometimes with a rusk. To support the falling powers of 
the stomach, a pill containing one grain of Cayenne pepper was taken every hour with some relief; after eleven or twelve hours it was discon. tinued. The painful and immoveable distension of the stomach from flatulence continued and even increased, and was particularly distressing in the nights, generally occurring, as well as the spasmodic cough and faintings, with unusual violence soon after midnight, and somewhat abating towards day-break. For the relief of this flatulence, peppermint-water, a small portion of brandy with hot water, and occasionally with half a glass of wine, were used; and on the recurrence of a disposition to faint, ammonia and sometimes æther were taken with advantage. But the most speedy and sensible relief under these extreme sinkings was produced by a musk draught, containing ten grains of that substance.

The first dose of this medicine appeared to diffuse its stimulating effects through the whole frame, exciting a sort of electric tingling, even to the extremities of the limbs, and an immediate feeling of renovated strength. The same effect was pro. duced in a somewhat less degree whenever it was repeated, and being left as a sort of sheet-anchor, it was taken four times afterwards in the course of the ten following days, when the most alarming paroxysms of faintness occurred.

On Friday the 14th, on giving up the Cayenne 
pepper, a warm decoction of bark was taken, with a view to support the powers of the stomach, which in a day or two was changed for a cold infusion of cinchona in lime water, which was at first more grateful to the stomach, but soon afterwards the extract in the form of pill was substituted for it. During the use of the cinchona the bowels became rather constipated, but were easily kept open by an occasional draught containing three drachms of tincture of senna, after enemata had failed. Beeftea, from the constant repetition, being now loathed by the stomach, sago milk, rice milk, and arrow. root were substituted, and still taken every hour night and day, the flatulence and cough being much increased if the intervals were prolonged. This plan was continued till Monday the 17th, when an urgent desire being expressed for animal food, a small quantity of roasted pheasant was al. lowed, but not repeated, in consequence of the oppression and increase of flatulence which it occasioned, and which was followed by an increased disturbance of the action of the heart. During the night and the whole of the following day, the disturbed and feeble state of the circulation was so great, that the extremities became cold, and required the constant application of bottles of hot water; and the feelings of languor and approach. ing dissolution were unusually distressing. At this time too, an œdema of the lower limbs, which had begun to shew itself a few days previously, 
also increased. The musk draught was then this afternoon, and with considerable relief to the feelings of languor, though the effect was less sensible and speedy than before.

Thursday, eoth. The enfeebled condition of the stomach, and especially the immoveable flatulence which was accompanied with a sensation of globus, being rather increased, as well as the ge neral debility, it was this day determined to give tip all solid food and medicine, and to limit the diet to asses' milk, to the extent of a quart daily, taken alternately every hour with cows' milk, into which a small quantity of tops and bottoms was occasionally broken. The benefit of this change in the ingesta was most sensible, for immediately the flatulence began to subside, and the harassing rough to diminish; and from this time a progressive, though very slow improvement of the strength and all the other symptoms took place. The stomach regained its power by very slow steps; this liquid diet was continued for about a week, when a fittle rice or sago was added to the milk; in a few days more, rice, bread, or light flour puddings was taken occasionally at some of the hourly meals; and on Monday the th of March, for the first time a little whiting was eateh, but produced im. frediately considerable oppression and flatelence, which was followed by a viotent beating of the hetrt, without irregularity, but at the ste of 160 
contractions in a minute, and which continued without intermission for a fortnight. It was romarkable, that during this period the arterial pulsations, which, as was before observed, were occasionally not synchronous with those of the heart, were now almost uniformly just half the number, the heart beating twice while the pulse in the extremities beat once. When this was not the case, the pulsations of the arteries were again different in number and strength from those of the heart. This disturbance of the digestion and of the other functions from the use of the fish, rendered it necessary to restrict the diet still to milk and puddings, and it was only by very slow and gradual steps that the stomach became able to receive more solid food, proceeding from pudding to eggs, and from these, in about ten days, to a small par. tion of chicken once in twenty-four hours, and after a few days more to a mutton-chop, scc. the first use of any article of diet more solid than the preceding being always attended with some op. pression and flatulence. It was also by the same slow progression that the stomach became capable of sustaining longer intervals of abstinence, acquiring gradually the power of fasting two hours, in the course of about three weeks from the commencement of the milk diet, but requiring tood in the night till the end of April. The stute of the circulation and the muscular strength have been still more slow in their progress towards ro- 
covery; the necessity of maintaining an erect pos: tare even during sleep, in consequence of the extreme disturbance of the action of the heart, and the sense of anxiety and oppression which the least inclination to the supine posture produced, remained six weeks, and six weeks more elapsed before a complete recumbent position could be resumed; and it was still longer before the power of lying on either side was regained, and before the pain and constriction in the region of the dia. phragm, which had been the source of the most severe sufferings in the earlier stages, were sufficiently removed to admit of a perfectly erect posture in walking. Even now, on the $22 \mathrm{~d}$ of July, the pulse has almost uniformly continued at the rate of 96, and the action of the heart is easily hurried, and even slightly disturbed in its regularity, by any sudden exertion or emotion. All the functions seemed to have regained their healthy condition, with the exception of the circulation and the muscular strength; weariness and aching of the limbs being produced by very small exer. tions in walking.

It may not, perhaps, detract from the authenticity of the details of this case to add, that it occurred in the person of the narrator, who, at the time of presenting these notes, Jan. 20th, 
1818, (one year after the attack,) continues slowly regaining his muscular strength, the circulation being hurried, and lassitude and aching of the limbs induced by very moderate exertions in walking, and the pulse at all times exceeding 80 beats in the minute. 